\title{
Insulators: linking genome architecture to gene regulation
} Robert AH White

Address: Department of Physiology, Development \& Neuroscience, University of Cambridge, Downing Street, Cambridge, CB2 3DY, UK

Email: rw108@cam.ac.uk

FI000 Biology Reports 2009, I:92 (doi: I0.34I0/BI-92)

The electronic version of this article is the complete one and can be found at: http://FI000.com/Reports/Biology/content/I/92

\begin{abstract}
Insulator elements have long been associated with a proposed domain boundary function, ensuring appropriate associations between regulatory elements and transcription units through the physical organisation of the genome into looped domains. Recent experiments in Drosophila have, however, highlighted a more direct involvement of insulators in transcriptional regulation.
\end{abstract}

\section{Introduction and context}

A major goal of genomic studies is to understand the regulatory architecture of the genome; how, for example, powerful regulatory elements, such as enhancers and silencers, which have the ability to act over large genomic distances, are constrained to act only on appropriate transcription units. There is considerable evidence for a domain model of genomic regulatory architecture where the actions of long-range regulatory elements are confined within chromosomal domains [1-3]. The promiscuity of enhancers is restricted to regulation of transcription units within the same domain. In this model of genome organisation, insulator elements are proposed to play a key role as domain gate-keepers, shielding genes within the domain from the influence of outside regulatory elements. How insulators function is still unclear but they have been proposed to associate together to form chromosomal loops providing a physical basis for chromosomal domains. Insulator elements have been shown to have appropriate activities for such a role; they are defined by their ability to block enhancer-promoter interaction and/or to provide boundaries between different chromatin states and have been demonstrated to be involved in chromosome looping. But does the segregation of the genome into domains through the formation of chromosomal loops really represent the endogenous function of insulator elements? Although this question has been asked in the past [4,5], recent results in Drosophila emphasising links between insulators and the regulation of transcription have once again brought it into focus.

\section{Major recent advances}

A number of proteins have been associated with insulator function in Drosophila, including the Suppressor of Hairywing $[\mathrm{Su}(\mathrm{Hw})]$ protein [6], CCCTC-binding factor (CTCF) [7], Centrosomal protein 190 (CP190) [8], Boundary element-associated factor (BEAF) [9], GAGA factor (GAF) [10] and Zeste-white-5 (Zw5) [11], and over the past few years, genomic binding sites for several insulator components have been mapped [12-18]. In support of the domain model, these studies reveal associations between particular insulator components and boundaries of chromatin state domains; for example, Bartkuhn et al. [14] document that CP190 and CTCF binding correlates strikingly with the boundaries of repressive chromatin domains marked by the H3K27me3 modification. This accords with genomic studies in vertebrates demonstrating that CTCF binding sites mark chromatin state boundaries $[19,20]$. However, the Drosophila genomic mapping experiments also reveal a strong association between particular insulator components and transcription start sites. This is especially true for some components, CP190, GAF and BEAF, but not for $\mathrm{Su}(\mathrm{Hw})$, suggesting that there may be distinct types of insulator complexes [15]. BEAF 32A and B were initially identified as factors binding to an insulator element (scs') associated with the 87A hsp70 heat shock locus [9]. Insulator activity is dependent on BEAF binding sites and BEAF is involved in the formation of a chromosomal loop through interaction with Zw5 bound to a second element (scs) [21]. However, despite its strong 'domain boundary' 
credentials, more than $85 \%$ of the 1820 BEAF binding sites in the genome are less than $300 \mathrm{bp}$ from transcription start sites [16]. Many (50\%) of these BEAF peaks are associated with head-to-head gene pairs, although these BEAF sites do not appear to function to insulate these adjacent promoter regions from each other. Rather, BEAF appears to be generally required to promote transcription. Sharing of components between insulators and promoters may fit with shared structural features of these elements (for example, both CTCF-binding sites and active transcription start sites are marked by the presence of specific histone variants and are associated with nucleosomes containing both the H3.3 and H2A.z variants [22]).

While this may be interpreted as indicating a link between insulators and transcriptional regulation, it might also suggest that 'insulator' components may serve a variety of functions and not all their binding sites are necessarily associated with insulator function. However, other experimental approaches provide support for a fundamental role for insulator-promoter interaction. It has been known for some time that some promoters can behave as insulators in enhancer-blocking assays [10]. However, it was never clear whether the mechanism of enhancer-block was the same in both cases. Recently, Chopra et al. [23] have uncovered a connection between promoters with enhancer-blocking activity and the presence of stalled RNA polymerase II (Pol II). Testing the ability of several Hox gene promoters to exhibit enhancer-blocking, promoters associated with stalled Pol II showed blocking function whereas several unstalled promoters did not. The enhancer-blocking activity was dependent on the function of the elongation factors Negative elongation factor (NELF) and DRB sensitivity-inducing factor (DSIF), which are thought to stabilise Pol II at the pause site. However, the key observation that draws together insulators and promoters is that the enhancer-blocking activity of two bithorax-complex insulators, Fab7 and Fab8, is also dependent on the elongation factors NELF and DSIF. As Fab7 and Fab8 do not seem to contain stalled polymerase, the implication is that the insulators interact with promoters to inhibit elongation. This fits with observations, using DamID (DNA adenine methyltransferase identification) or chromosomal conformation capture, that the Fab7 and Fab8 insulators can form loops that bring them into contact with promoter regions of the $A b d-B$ Hox gene and that this configuration is associated with repression of $A b d-B$ transcription $[24,25]$.

In support of the generality of interaction between insulators and the regulation of transcription elongation, Jiang et al. [16] found considerable overlap between BEAF and NELF binding sites. In addition, vertebrate studies using chromatin topology assays have identified insulator-promoter associations at several loci, including Igf2-H19 [26,27] and CFTR [28].

\section{Future directions}

Overall, the above combination of genomic binding site location and functional analysis currently indicates a more intimate relationship between insulators and the regulation of gene transcription than is suggested by the domain boundary/chromosome loop model. Insulators may serve a diverse range of functions; as classical boundaries, as boundaries of regulatory regions $[13,29]$, and as regulators of transcription. Even the regulatory effects of insulators can apparently be diverse; BEAF appears to be predominantly required to facilitate transcription whereas the above interactions between the $F a b$ insulators and $A b d-B$ promoters occur in a repressive context. Is there a common thread in this diversity? It is feasible that insulator elements in general are involved in the specification of chromosomal topology; this could provide a common function but, depending on the context, could have diverse consequences for chromatin state and transcriptional regulation.

\section{Abbreviations}

BEAF, Boundary element-associated factor; CP190, Centrosomal protein 190; CTCF, CCCTC-binding factor; DamID, DNA adenine methyltransferase identification; DRB, 5,6-dichloro-1- $\beta$-D-ribofuranosylbenzimidazole; DSIF, DRB sensitivity-inducing factor; GAF, GAGA factor; NELF, Negative elongation factor; $\mathrm{Su}(\mathrm{Hw})$, Suppressor of Hairy-wing; Pol II, RNA polymerase II; Zw5, Zeste-white-5.

\section{Competing interests}

The author declares that he has no competing interests.

\section{Acknowledgments}

RAHW acknowledges funding from the Biotechnology and Biological Sciences Research Council, the Newton Trust and the National Institutes of Health.

\section{References}

I. Labrador M, Corces VG: Setting the boundaries of chromatin domains and nuclear organization. Cell 2002, I I I:I5I-4.

2. Wallace JA, Felsenfeld G: We gather together: insulators and genome organization. Curr Opin Genet Dev 2007, I 7:400-7.

3. de Wit E, van Steensel B: Chromatin domains in higher eukaryotes: insights from genome-wide mapping studies. Chromosoma 2009, I I 8:25-36.

4. Cai H, Levine $M$ : Modulation of enhancer-promoter interactions by insulators in the Drosophila embryo. Nature 1995, 376:533-6.

5. Geyer PK: The role of insulator elements in defining domains of gene expression. Curr Opin Genet Dev 1997, 7:242-8.

6. Geyer PK, Corces VG: DNA position-specific repression of transcription by a Drosophila zinc finger protein. Genes Dev 1992, 6:1865-73. 
7. Moon H, Filippova G, Loukinov D, Pugacheva E, Chen Q, Smith ST, Munhall A, Grewe B, Bartkuhn M, Arnold R, Burke LJ, RenkawitzPohl R, Ohlsson R, Zhou J, Renkawitz R, Lobanenkov V: CTCF is conserved from Drosophila to humans and confers enhancer blocking of the Fab-8 insulator. EMBO Rep 2005, 6:165-70.

8. Pai CY, Lei EP, Ghosh D, Corces VG: The centrosomal protein CPI 90 is a component of the gypsy chromatin insulator. Mol Cell 2004, I6:737-48.

9. Zhao K, Hart CM, Laemmli UK: Visualization of chromosomal domains with boundary element-associated factor BEAF-32. Cell 1995, 81:879-89.

10. Ohtsuki S, Levine M: GAGA mediates the enhancer blocking activity of the eve promoter in the Drosophila embryo. Genes Dev 1998, I 2:3325-30.

II. Gaszner M, Vazquez J, Schedl P: The Zw5 protein, a component of the scs chromatin domain boundary, is able to block enhancer-promoter interaction. Genes Dev 1999, 13:2098-107.

12. Adryan B, Woerfel G, Birch-Machin I, Gao S, Quick M, Meadows L, Russell S, White R: Genomic mapping of Suppressor of Hairywing binding sites in Drosophila. Genome Biol 2007, 8:RI67.

13. Holohan EE, Kwong C, Adryan B, Bartkuhn M, Herold M, Renkawitz R, Russell S, White R: CTCF genomic binding sites in Drosophila and the organisation of the bithorax complex. PLoS Genet 2007, 3:el I 2.

14. Bartkuhn M, Straub T, Herold M, Herrmann M, Rathke C, Saumweber H, Gilfillan GD, Becker PB, Renkawitz R: Active promoters and insulators are marked by the centrosomal protein 190. EMBO J 2009, 28:877-88.

15. Bushey AM, Ramos E, Corces VG: Three subclasses of a Drosophila insulator show distinct and cell type-specific genomic distributions. Genes Dev 2009, 23:1338-50.

16. Jiang N, Emberly E, Cuvier O, Hart CM: Genome-wide mapping of boundary element-associated factor (BEAF) binding sites in Drosophila melanogaster links BEAF to transcription. Mol Cell Biol 2009, 29:3556-68.

17. Smith ST, Wickramasinghe P, Olson A, Loukinov D, Lin L, Deng J, Xiong Y, Rux J, Sachidanandam R, Sun H, Lobanenkov V, Zhou J: Genome wide ChIP-chip analyses reveal important roles for CTCF in Drosophila genome organization. Dev Biol 2009, 328:5। 8-28.

18. The National Human Genome Research Institute (NHGRI) model organism ENCyclopedia Of DNA Elements (modENCODE). [http://www.modencode.org/].

19. Barski A, Cuddapah S, Cui K, Roh TY, Schones DE, Wang Z, Wei G, Chepelev I, Zhao K: High-resolution profiling of histone methylations in the human genome. Cell 2007, 129:823-37.

FI000 Factor 7.0 Must Read

Evaluated by Steven Henikoff 22 May 2007, Xing Wang Deng 05 Jun 2007, Michael Meisterernst 19 Jun 2007, Deyou Zheng 29 Jun 2007, Magdalena Zernicka-Goetz 15 Jan 2008
20. Cuddapah S, Jothi R, Schones DE, Roh TY, Cui K, Zhao K: Global analysis of the insulator binding protein CTCF in chromatin barrier regions reveals demarcation of active and repressive domains. Genome Res 2009, 19:24-32.

FI000 Factor 6.0 Must Read

Evaluated by I King Jordan 10 Feb 2009

21. Blanton J, Gaszner M, Schedl P: Protein:protein interactions and the pairing of boundary elements in vivo. Genes Dev 2003, 17:664-75.

FI000 Factor 3.0 Recommended

Evaluated by Wendy Bickmore 17 Dec 2003

22. Jin C, Zang C, Wei G, Cui K, Peng W, Zhao K, Felsenfeld G: H3.3/ H2A.Z double variant-containing nucleosomes mark 'nucleosome-free regions' of active promoters and other regulatory regions. Nat Genet 2009, 41:94I-5.

23. Chopra VS, Cande J, Hong JW, Levine M: Stalled Hox promoters as chromosomal boundaries. Genes Dev 2009, 23:1505-9.

FI000 Factor 6.0 Must Read

Evaluated by Pamela Geyer 20 Jul 2009

24. Cleard F, Moshkin Y, Karch F, Maeda RK: Probing long-distance regulatory interactions in the Drosophila melanogaster bithorax complex using Dam identification. Nat Genet 2006, 38:93I-5.

FI000 Factor 3.2 Recommended

Evaluated by Bruce Morgan 08 Aug 2006, Leonie Ringrose 14 Aug 2006

25. Lanzuolo C, Roure V, Dekker J, Bantignies F, Orlando V: Polycomb response elements mediate the formation of chromosome higher-order structures in the bithorax complex. Nat Cell Biol 2007, 9: $1167-74$

FI000 Factor 3.2 Recommended

Evaluated by Rob White 0 I Oct 2007, Leonie Ringrose 3 I Oct 2007

26. Yoon YS, Jeong S, Rong Q, Park KY, Chung JH, Pfeifer K: Analysis of the HI9ICR insulator. Mol Cell Biol 2007, 27:3499-5IO.

27. Li T, Hu JF, Qiu X, Ling J, Chen H, Wang S, Hou A, Vu TH, Hoffman AR: CTCF regulates allelic expression of Igf2 by orchestrating a promoter-polycomb repressive complex 2 intrachromosomal loop. Mol Cell Biol 2008, 28:6473-82.

28. Blackledge NP, Ott CJ, Gillen AE, Harris A: An insulator element $3^{\prime}$ to the CFTR gene binds CTCF and reveals an active chromatin hub in primary cells. Nucleic Acids Res 2009, 37:1086-94.

29. Maeda RK, Karch F: The ABC of the BX-C: the bithorax complex explained. Development 2006, I33:14|3-22. 